\title{
LES ARTHROPODES VECTEURS DE LA PESTE BUBONIQUE
}

\author{
Par A.-G. CHABAUD
}

De 1907 à 1912, la Commission des Indes avait établi que la peste bubonique était essentiellement une affection des rats domestiques transmise à l'homme par la puce de ces animaux, Xenopsylla cheopis.

Le problème apparaît aujourd'hui beaucoup plus complexe, car deux ordres de faits nouveaux sont venus compléter la conception première. D'une part, la mise en évidence de la peste selvatique, d'autre part, la démonstration d'une peste bubonique interhumaine, sans passage par les rongeurs, les puces s'infectant directement sur l'homme.

Il est donc possible de schématiser l'épidémiologie de la peste par trois cycles distincts :

$1^{\circ}$ Chez les rongeurs sauvages.

$2^{\circ}$ Chez les rongeurs domestiques.

$3^{\circ}$ Chez l'homme.

Dans chacun de ces cycles, les puces vectrices seront différentes. En outre, le passage d'un cycle à l'autre ne pourra se faire que par des vecteurs ayant une spécificité parasitaire assez large et les puces les plus importantes dans ce cas ne seront pas fatalement celles qui jouent le plus grand rôle à l'intérieur d'un même cycle.

Nous avons cherché, en suivant ce plan, à faire une liste des arthropodes étudiés expérimentalement dans la transmission de la peste et tenté d'indiquer l'importance relative de chacun pour chaque cycle considéré, et dans le passage d'un cycle à l'autre.

\section{CYCLE SELVATIQUE}

Il est très difficile de connaître l'importance relative de chaque puce, car chacune est capable de transmettre la peste. On peut toujours comparer expérimentalement deux espèces, mais le résul-

Anv. de Parasitologie, T. XXII, Nos $3-4,1947$, p. 169-200. 
tat de la comparaison n'est qu'un des éléments du problème. En effet, l'épidémiologiste chargé d'une enquête sur un foyer de peste selvatique est astreint à l'étude d'un grand nombre de détails purement locaux et, par là même, très difficiles à codifier : les variations de température et d'humidité de la région, les mœurs des rongeurs, l'intensité et la nature de leur parasitisme, les trajets habituels de leurs migrations et les rapports qu'ils peuvent contracter avec l'homme, nécessitent, dans tous les cas, une étude spéciale, car chaque région possède fatalement ses particularités.

Devignat (1946) a cherché à résoudre ces difficultés en prenant pour exemple la peste du Lac Albert, au Congo Belge.

Il envisage six modes de transmission :

inter-domestique, selvo-domestique, inter-selvatique, selvo-humain, inter-humain, domestico-humain.

Un Index commun de transmissibilité, Q, est calculé tous les ans, de façon parallèle, pour chacun des six modes de transmission (Q1, Q2..., Q6). Cet index est donné par la formule générale :

$$
\mathrm{Q}=\mathrm{K}\left(k(i+t)+k^{\prime}\left(i^{\prime}+t^{\prime}\right)+k^{\prime \prime}\left(i^{\prime \prime}+t^{\prime \prime}\right) \ldots . .\right)
$$

Les quatre variables représentent respectivement :

$\mathrm{K}=$ Constante de réceptivité : facteur de réduction dérivant de la réceptivité des mammifères composant l'un ou l'autre plan. C'est une moyenne calculée par une formule tenant compte à la fois des taux de capture des diverses espèces et de leur réceptivité respective (en $0 / 0)$ à l'inoculation d'une dose virulente standard. L'expérience semble prouver que $\mathrm{K}$ n'est pas constant mais variable avec l'année, la saison ou la localité.

$i=$ Index puce-rat et $t=I n d e x-p u c e-t e r r i e r$ représentant la fréquence de l'insecte, sont établis sur un nombre assez large de recherches (100 rongeurs pour $i, 10$ terriers pour $t$ ).

$k=$ Constante de vection : facteur de réduction dépendant de la qualité vectrice de l'espèce placée dans les conditions optimales de vection.

Cette constante de vection ne peut pas être basée sur le $0 / 0$ de décès d'animaux mis en présence des puces infectées, car pratiquement, ou bien tous les rongeurs meurent, ou bien, tous sont indemnes, et le coefficient est égal à 1 ou à 0 . C'est pourquoi Devignat a imaginé un procédé plus délicat, basé sur la mortalité par rapport au temps. Il établit une courbe avec en abscisse le jour de l'expérience, et en ordonnée le chiffre cumulatif (exprimé en $0 / 0$ ) des décès de rongeurs à chaque jour de l'expérience. Il suffit ensuite de considérer la surface limitée par la courbe et la ligne des abscisses 
pour avoir un chiffre variable avec l'espèce ; la même opération a été faite après l'inoculation d'une souche standard : les surfaces trouvées pour les puces sont rapportées à la surface donnée par la souche standard.

L'auteur reconnaît que l'ensemble de la méthode donne lieu à quelques critiques. Il admet, en particulier, ne pas tenir compte de la fréquence des contacts entre les rongeurs d'un mème plan.

D'autre part, on estime ici que les espèces pulicidiennes trouvées sur un rongeur donné dépendant surtout du modus vivendi (selvatique ou domestique) de celui-ci et non de l'espèce parasite en cause, l'auteur peut ainsi prendre en bloc, d'une part, la constante de réceptivité des rongeurs $\mathrm{K}$, et d'autre part, les facteurs dépendant des puces.

Cette simplification, si elle est valable pour le Congo Belge, est peut-être plus difficile à admettre dans d'autres régions.

Quoi qu'il en soit, cet important travail donne une base précise qui sera très précieuse lorsque sa valeur générale aura été précisée dans d'autres régions.

Nous nous plaçons ici à un point de vue moins général, ne considérant que les facteurs liés à l'espèce vectrice.

L'arthropode peut être étudié à différents points de vue soit :

A) Comme vecteur entre rongeurs de la même espèce. Les points les plus intéressants seront :

$1^{\circ}$ La fréquence sur l'hôte aux différentes époques de l'année.

$2^{\circ}$ La facilité d'infestation de la puce sur le rongeur pesteux : le $0 / 0$ de puces infectées spontanément donne ici une notion très utile.

$3^{\circ}$ La facilité de transmission du germe de la puce au rongeur sain (Méthode de Devignat).

$4^{\circ}$ Le nombre d'animaux pouvant être infectés par une puce (Wheeler et Douglas).

$5^{\circ}$ La durée de la période d'infection de la puce.

$6^{\circ}$ La biologie de l'insecte : sa longévité, sa résistance aux différentes conditions de chaleur et d'humidité, le nombre moyen des repas, le mode de parasitisme, soit permanent, sur le même hôte, soit intermittent, la puce quittant un premier rongeur pour rester dans le terrier et pouvant ensuite s'attaquer à un nouvel hôte.

B) Comme vecteur de la peste entre hôtes appartenant à des espèces différentes et que nous appellerons hôte $\mathrm{A}$ et hôte $\mathrm{B}$.

Il est alors nécessaire de noter :

$1^{\circ}$ La fréquence respective sur l'hôte A et sur l'hôte B.

$2^{\circ}$ La facilité d'infestation sur l'hôte A. 
$3^{\circ}$ La facilité de transmission à l'hôte $\mathrm{B}$.

$4^{\circ}$ Le nombre d'animaux $B$ pouvant être infectés expérimentalement par une même puce.

$5^{\circ}$ La durée de la période d'infection de la puce.

$6^{\circ}$ La biologie de la puce et en particulier la facilité avec laquelle elle passe de l'hôte A à l'hôte B.

Les renseignements obtenus dans la première étude (vecteurs entre rongeurs de la même espèce) sont donc largement utilisables dans la seconde partie (vecteur d'un hôte $\mathrm{A}$ à un hôte $\mathrm{B}$ ).

Wheeler et Douglas (1941) ont cherché à exprimer certains de ces facteurs d'une façon numérique. Ils définissent différents rapports dont le produit donne un chiffre traduisant, pour une part tout au moins, le danger que présente chaque espèce. Ils établissent, par des études individuelles :

Potentiel d'infection, ou rapport du nombre de puces qui s'infectent sur le nombre de puces ayant pris le repas infectant.

Potentiel vecteur, ou rapport du nombre de puces qui transmettent l'infection au nombre de puces infectées.

Potentiel de transmission, ou nombre de transmissions, c'est-à-dire chiffre moyen de rongeurs infectés pour une puce infectieuse.

Le produit de ces facteurs représente le nombre de transmissions effectuées par un individu moyen ou efficience du vecteur.

Voici, par exemple, les valeurs obtenues pour Ceratophyllus montanus et $X$. cheopis :

\begin{tabular}{|l|c|c|c|c|}
\hline & Pot. INF. & Pot. vect. & Pot. Tr. & EFF. vect. \\
\hline C. montanus...... & 0,85 & 0,52 & 2,58 & $=1,14$ \\
X. cheopis........ & 0,98 & 0,29 & 1,44 & $=0,39$ \\
\hline
\end{tabular}

Des résultats plus récents (1945) ont modifié ces premiers chiffres.

X. cheopis $=0,43, C$. montanus $=0,84$. Enfin, Ctenocephalides felis et Hoplopsyllus anomalus ont tous les deux le coefficient 0 . Ce chiffre peut paraître surprenant, puisque les mêmes auteurs ont réussi (1941), avec la première espèce, à transmettre la peste à l'animal d'expérience qui était dans les deux cas la souris blanche. 
D'autre part, une cause d'erreur semble difficile à éviter lorsque le mammifère pris comme test n'est pas l'hôte préféré de la puce.

Cependant, la méthode a l'avantage de fixer, pour chaque espèce, un chiffre de base susceptible de discussions ultérieures, et capable d'acquérir ainsi une réelle précision.

\section{Afrique}

La peste ancienne d'Afrique, définie par R、JoRge (1935), ne semble pas avoir été accompagnée de foyers selvatiques. La peste contemporaine, qui débute en 1900 , a fait le tour de l'Afrique en restant, elle aussi, essentiellement côtière. Elle a pu cependant pénétrer en quelques régions et s'y maintenir avec une gravité variable mais, en général, assez faible.

Nous passerons rapidement en revue ces pestes continentales, pour traiter plus spécialement le foyer de l'Afrique du Sud, exemple typique de la conversion de la peste commune en peste selvatique.

\section{A. - Haute-Egypte}

Certains rongeurs sauvages de la Haute-Egypte sont atteints de peste, mais il s'agit bien davantage d'une « peste d'accompagnement 》 des rats domestiques que d'une peste selvatique proprement dite. Deux espèces ont été trouvées infectées spontanément : Acomys cahirinus, surtout rural, mais aussi domestique, qui est l'hôte normal de $X$. chephrenis ; Arvicanthis niloticus, muridé également sylvestre, mais qui peut se rencontrer dans les habitations. La peste de cette région semble très efficacement combattue par Mustela africana, petit viverridé très abondant, qui détruit ces rongeurs et même Rattus rattus.

$X$. cheopis est fréquent en Haute-Egypte, les autres espèces pulicidiennes, probablement d'intérêt secondaire, ne paraissent pas avoir fait l'objet d'études spéciales.

\section{B. - TUNisie}

Les puces et les rongeurs ont été catalogués par Wassilief (1931 et 1932). Il a remarqué que le mérion (Meriones shawi) est surtout répandu au centre de la Tunisie, c'est-à-dire dans la région la plus contaminée par la peste. Par un hasard heureux, ce mammifère est peu sensible à l'infection. Dans l'extrême sud, Psam- 
momys roudairei est très abondant et très sensible à la maladie, mais le secteur n'est pas atteint par l'épidémie, et il n'y a pas, actuellement, de peste selvatique en Tunisie. Cependant une épidémie qui a envahi, en 1920 , le centre du pays, était peut-être, sans qu'on en ait de preuves certaines, secondaire à une migration de Gerbilles venues de Tripolitaine. Les espèces Gerbillus hirtipes et G. eatoni latestei font partie de la faune tunisienne.

\section{C. - Maroc}

En 1911, un foyer s'est manifesté à l'intérieur du pays, dans les régions des Doukkala et de la Chaouia. Il est possible que les rongeurs sauvages aient été à son origine, mais il y a très peu de renseignement à cet égard.

\section{D. - Afrique Occidentale}

La faune des rongeurs a été étudiée par Cazanove (1929) et Lefrou (1932). Les espèces sauvages les plus remarquables sont :

Mastomys coucha dont la distribution, très large, couvre toute la moitié sud de l'Afrique (voir ci-dessous). Arvicanthus rufinus, également très abondant. Golunda campana a été trouvé fréquemment infecté par Cazanove (1929), ainsi que Cricetomys gambianus. La musaraigne Crocidura stampfii fréquente les habitations (Léger et Baury, 1923). Nous pouvons encore citer Xerus erythropus. Toutes ces espèces sont atteintes spontanément par la peste. Plus rarement les chacals, les lièvres (Lefrou, 1929, au Cayor), les hérissons ont été trouvés infectés.

La peste est donc ici largement répandue chez les rongeurs sauvages, cependant R. Jorge se refuse à parler de peste selvatique dans cette région, car, dit-il, " le rat s'en mêle, et, même, est à la tête de l'épidémisation ».

Les puces le plus souvent rencontrées sont $X$. cheopis, Echidnophaga gallinacea, X. astia, X. brasiliensis, Synosternus pallidus, Ctenophalides felix et $C$. canis.

$S$. pallidus est l'espèce la plus intéressante de la région à cause de sa très grande fréquence dans les habitations (Roubaud, 1931). Elle a une large distribution géographique : Egypte, Soudan égyptien, Tunisie ; elle est même connue au Brésil et en Russie. L'espèce a peu de spécificité parasitaire, puisqu'elle se trouve sur l'homme, le chien, les renards, les hyènes, les hérissons et différents rongeurs. Son rôle pestigène, encore mal connu, sera envisagé en même temps que celui des autres puces vectrices inter-humaines. 


\section{E. - CONGo Belge}

Un foyer existe à la frontière de l'Ouganda, dans la région d'Ituri. L'étude de cette zone a fait l'objet du travail précédemment cité de Devignat.

Le rongeur le plus important est encore Mastomys coucha ugandæ. L'auteur le considère comme franchement domestique.

Arvicanthis abyssinicus se rencontre souvent dans les habitations. La liste complète des rongeurs est donnée par Devignat. Les espèces trouvées infectées spontanément sont avec Mastomys coucha.

A. abyssinicus $\ldots \ldots \ldots \ldots \ldots \ldots \ldots, 1,4$ cas pour 1.000

Lophuromys aquilus .............. 3,4 - -

Lemniscomys striatus ............ 6,1 - - -

Otomys tropicalis $\ldots \ldots \ldots \ldots \ldots \ldots .6,0,6-$

Dasymys bentleyæ $\ldots \ldots \ldots \ldots \ldots \ldots .0,7$ -

Les autres espèces sont moins fréquentes ou moins souvent infectées. La constance $k$ de réceptivité a été recherchée pour les rongeurs les plus importants.

Du point de vue entomologique, 15 espèces de puces ont été relevées ; les espèces pestifères sont données avec un chiffre indiquant le rapport du nombre de souches isolées au nombre d'exemplaires de puces inoculées :

$X$. cheopis $(1,10 / 0), X$. brasiliensis $(0,40 / 0)$, Dincpsyllus lypusus $(0,30 / 0)$, Ctenophtalmus sp. $(0,30 / 0)(1)$, Ctenocephalides felis strongylus $(0,20 / 0)$, Leptosylla rthiopica $(2,20 / 0)$.

Le chiffre donné pour L. æthiopica peut induire en erreur car l'espèce est assez localisée et l'étude n'a pu être faite qu dans une seule zone qui était précisément un foyer pesteux.

Des expériences ont été entreprises pour déterminer le caractère pestigère des différentes espèces. Les résultats se résument ainsi :

Ctenocephalides felis strongylus est très intéressante, car c'est la seule espèce qui vive de façon élective sur l'homme, mais elle n'a pas transmis le germe à la souris blanche, au cours de plusieurs expériences.

Ctenophtalmus sp. a, elle aussi, expérimentalement, un coefficient 0 .

(1) Les Ctenophtalmus identifiés dans la région sont C. evidens, C. cabirus, C.. devignati et $C$. phyris. 
Mais trois espèces se sont montrées bonnes vectrices ; ce sont : $X$. cheopis $0,96, X$. brasiliensis $0,71, D$. lypusus 0,90 .

L'auteur tire alors les conclusions suivantes :

La peste du Lac Albert trouve des conditions très favorables sur le plan selvatique. La transmission se faisant essentiellement par D. lypusus. Cette espèce est aussi la plus importante pour le passage de la peste selvatique au rat de hutte Mastomys coucha, et éventuellement à l'homme.

$X$. cheopis et $X$. brasiliensis se partagent le rôle de vecteurs entre rats de hutte et peuvent aussi, accidentellement, faire le passage, soit sur le plan selvatique, soit, plus exceptionnellement, à l'homme. La peste reste rare pour l'homme grâce au peu d'efficacité de $C$. felis strongylus.

\section{F. - Afrique de L'Est}

L'ensemble de l'Afrique Orientale s'oppose à l'Afrique du Sud en ce que la peste y sévit chez les rongeurs domestiques plutôt que chez les rongeurs sauvages.

Les espèces que nous pouvons citer sont donc plutôt des victimes accidentelles de la peste domestique que les principaux réservoirs de virus.

Les deux espèces domestiques prédominantes sont Mastomys coucha et le rat noir sous ses diverses races : Rattus rattus kijabius et $R$. rattus alexandrinus. Elles forment un foyer indépendant des gerbilles.

Le rat noir étend actuellement son aire de dispersion aux dépens de $M$. coucha, mais les deux espèces sont également dangereuses.

Arvicanthus abyssinicus tient la troisième place. Citons accessoirement, parce que trouvés spontanément infectés : Lemniscomys massaicus en Ouganda et Kenya. Golunda fallax et Mus dolichurus au Kilima-Ndjaro. Cricetomys gambianus en Mozambique.

Les puces vectrices les plus importantes sont $X$. cheopis et $X$. brasiliensis. La première prédomine dans les maisons des villes, brasiliensis serait plus rurale, se rencontrant plutôt dans les huttes indigènes.

Dinopsyllus lypusus sera étudiée avec l'Afrique du Sud. Les espèces secondaires sont : E. gallinacea et Ctenocephalides.

Quelques espèces sont plus spéciales à la région qui nous occupe. $X$. bantorum, recueillie sur Mus norvegicus en Ouganda, a été décrite par Jordan (1938). Son rôle n'est pas connu, car elle avait probablement été confondue par X. cheopis. 
$X$. syngenis trouvée par Lamborn (1940) sur des rongeurs en zone infectée dans le Nyassaland.

Ctenophtalmus cabirus est une puce signalée sur Arvicanthis $s p$. au Kenya et sur Rattus en Afrique Orientale. Symes (1932), le premier, a démontré que l'espèce est un vecteur expérimental ; effectivement, depuis cette découverte, la transmission a pu être réalisée avec cinq exemplaires seulement, mais Symes et Roberts (1934) n'ont jamais trouvé d'exemplaires spontanément infectés à Nairobi.

\section{G. - Afrigue du Sud}

C'est aux environs de 1900 que la peste a été introduite en Afrique du Sud ; localisée au début dans les ports, sur les rongeurs domestiques, l'infection s'est éteinte chez ceux-ci, mais les rongeurs sauvages avaient été contaminés et l'épizootie s'est installée chez ces derniers de façon permanente et couvre maintenant une très vaste surface.

Mitchell, Pirie et Ingram (1927) ont fait une étude qui a pu être prise comme type dans les différentes pestes selvatiques reconnues ultérieurement. De nouvelles publications (De Meillon, Davis...) ont complété les premières acquisitions. Douze espèces de mammifères ont été trouvées naturellement infectées ; nous en donnons la liste ici :

1. La gerbille Tatera logengulæ semble le principal vecteur. C'est en effet l'espèce la plus fréquente et ses mœurs permettent une grande dissémination de l'épizootie ; elle vit en famille de $4^{\circ}$ à 24 et a tendance à déplacer son terrier et à en visiter d'autres ; les ectoparasites trouvés sur elle sont :

Dinopsyllus lypusus, Chiastopsylla rossi, Xenopsylla eridos, Listropsylla stygius, Ctenophtalmus calcatus, Leptosylla musculi, $X$. hipponax, un anoploure - Linognathoides faurei - et quatre acariens.

2. Le rat multimamellé Mastomys coucha présente aussi un intérêt de premier ordre, car c'est probablement lui le plus dangereux pour l'homme. Très abondant partout, il loge aussi bien dans les terriers en plein champ que dans le sol ou le toit des huttes. D'une extrémité à l'autre de son immense zone de dispersion, c'est lui le principal intermédiaire entre rongeurs sauvages et rongeurs domestiques, et lorsqu'il n'y a pas de peste selvatique, il peut, à lui seul ou associé à Rattus rattus, entretenir une peste domestique indépendante des Gerbilles. Ses ectoparasites sont en Afrique du Sud :

Dinopsyllus lypusus, Xenopsylla eridos, Chiastopsylla rossi, 
$X$. brasiliensis, Pulex irritans, un pou - Linognatoides faurei - et un acarien - Lælaps muricola.

En dehors de l'Afrique du Sud, on a aussi trouvé sur lui :

$X$. cheopis, Cenophtalmus sp. et Letopsylla æthiopica.

3. Desmodillus auricularis est une gerbille qui, comme Tatera lobengulæ, vit en petits groupes, mais elle est moins migratrice et pour cette raison est probablement moins dangereuse bien qu'elle soit communément infectée de puces : $X$. eridos et Dinopsyllus lypusus.

4. Geosciurus capensis vit aussi en famille dans de grands terriers souvent en association étroite avec les gerbilles. Il est l'hôte de :

Xenopsylla erilli, Ctenocephalus canis, Echidnophaga bradyta, E. gallinaceus, X. eridos, Dinopsyllus lypusus, Pulex irritans, le pou L. faurei, et un acarien, Hæmolælaps sp.

5. Rhabdomys pumilio, la " souris rayée 》, construit des terriers à la surface du sol, où elle vit en famille de 12 à 30 . Elle se trouve aussi dans les grains, les fourrages, et adopte facilement les terriers de gerbilles ou de Mastomys coucha. Les puces trouvées sur elle sont :

D. lypusus, X. eridos, C. rossi, Listropsylla agrippinæ, L. cheluræ, Chiastopsylla octavii, Dinopsyllus longifrons.

6. Malacothrix typicus, contrairement aux précédents, n'a pas l'habitude de visiter les terriers voisins, mais il est capable de s'éloígner à de grandes distances de son habitat ; il est l'hôte de :

D. lipusus, X. eridos, Listropsylla stygius.

7. Pedetes caffer, le "Springhare 》, est assez rarement atteint spontanément. Il pourrait cependant être responsable de foyers isolés qui s'observent dans la propagation de la peste selvatique, car il parcourt de grandes distances. Une seule espèce pulicidienne a été identifiée sur lui, c'est $X$. caffer. Les espèces suivantes paraissent avoir un rôle plus effacé ; elles sont citées avec la liste de leurs ectoparasites :

8. Paratomys luteolus : Chiastopsylla pitchfordi, L. agrippinæe, D. longifrons, X. eridos, C. rossi.

9. Myotomys broomi : Chiastopsylla mulleri, L. agrippinx, C. rossi, X. eridos, Hypsophtalmus aganippes.

10 Lepus saxatilis et

11. L. zuluensis, dont les parasites ne sont pas connus.

Les arthropodes de ces différents mammifères, autres que les siphonaptères, ont refusé de se nourrir sur l'homme, mais ils ont 
peut-être leur part dans la transmission entre rongeurs. En effet, les acariens sont nombreux dans les terriers des gerbilles et sont plus résistants que les puces aux conditions extérieures.

Parmi les siphonaptères les plus intéressants nous avons :

1. Dinopsyllus lypusus (J. et R.), vecteur expérimental entre gerbilles. A jeun depuis deux ou trois jours, il se nourrit sur l'homme. Il se rencontre toute l'année dans une grande partie de l'Afrique méridionale et orientale et à Madagascar.

2. Chiastopsylla rossi (Watrst.), espèce aussi fréquente que la précédente sur les gerbilles et sur Mastomys coucha, et qui a donné expérimentalement des résultats très comparables. Elle est capable de transmettre la peste de rongeurs artificiellement infectés à des animaux sains, les espèces utilisées étant les gerbilles et Mastomys coucha. A jeun depuis deux jours, elle pique l'homme. Cette espèce est plus fréquente pendant la saison sèche. La survie a été de 27 jours, expérimentalement, mais elle est probablement beaucoup plus longue dans les terriers.

3. $X$. eridos (Roths.), étudiée dans les mêmes conditions. C'est aussi un vecteur expérimental. Elle pique l'homme mais plus difficilement, elle est fréquente sur Parotomys brantsi. De Meillon (1942) remarque que la zone où $X$. eridos est la plus commune est celle dans laquelle la peste semble le plus fermement établie. (Centre et Nord de l'Etat d'Orange et partie méridionale du Transvaal).

4. X. hirsuta (Ing.), démontrée à Johannesburg (Depart. of Entomology 1930), capable d'effectuer la transmission de rongeur à rongeur.

5. X. piriei (Ing.), parasite de Desmodillus auricularis ; est connue pour être associée à la peste.

6. $X$. erilli (Roths.), citée comme pouvant piquer l'homme.

7. $X$. brasiliensis, qui peut se trouver sur des rongeurs selvatiques, mais elle est beaucoup plus un parasite de rongeurs domestiques ou semi-domestiques tels que Mastomys coucha.

\section{H. - Madagascar}

L'épidémiologie de Madagascar pourrait être rapprochée de celle des Indes.

Le rat noir ou ses variétés sont presque exclusivement en cause. Cependant, en 1933, Brachytarsomya albicaudata, rongeur sylvestre, serait sorti de la forêt pour envahir les régions avoisinantes. Les indigènes auraient remarqué une grande mortalité chez le rongeur et, par la suite, une dizaine de cas de peste bubonique chez l'hom- 
me (Sorel, 1937). Ces constatations n'ont jamais pu être vérifiées et on n'a pas la preuve d'une infection spontanée ; mais le rongeur est expérimentalement très sensible à la peste.

Les siphonaptères les plus intéressants à Madagascar sont :

$X$. cheopis très répandue dans les habitations.

Synopsyllus fonquernii est une puce de hérissons qui, récemment, s'est beaucoup multipliée, et devient commune sur le rat domestique. Elle est capable de transmettre expérimentalement la peste par piqûre (Sorel, 1937) et la découverte d'exemplaires spontanément infectés n'est pas exceptionnelle. D'après Girard (1942) on la rencontre surtout en saison froide, en dehors des habitations et des terriers de rats ; mais Le Gall (1943) trouve cependant que $200 / 0$ des puces récoltées dans les habitations appartiennent à cette espèce.

E. gallinacea, Leptosylla musculi, D. lypusus, C. felis, P. irritans semblent peu fréquentes dans les statistiques (Le Gall, 1943). Absence complète de $C$. fasciatus.

\section{Amérique du Nord}

La peste selvatique semble s'être installée en 1906 par contact entre les rats domestiques de San Francisco et les sciuridés de la zone avoisinante. L'épizootie selvatique atteint les Montagnes Rocheuses (1937). En 1939, elle s'étend depuis la Montana jusqu'au Sud de l'Etat de New Mexico, et en 1944, elle a gagné les grandes plaines des Etats de Dakota, de Montana, de Wyoming, de Culorado, de New Mexico et d'Oklahoma (Craig et Faust, 1945).

Eskey fait remarquer que les Citelles semblent être les principaux responsables de l'étendue de l'épizootie. Les différentes espèces ont, en effet, une distribution très large et leurs instincts grégaires favorisent la diffusion de la peste. De nombreuses espèces ont été trouvées spontanément infectées. Elles seront énumérées dans un tableau général en même temps que les puces qu'elles hébergent. Les marmottes, les chiens de prairies, les Eutamias participent à l'épizootie.

Une mention spéciale peut être faite pour le «burrowing owl », Speotyto cunicularia. Ce hibou se nourrit de Citelles morts qu'il transporte dans son terrier. Au cours d'une épizootie de Citellus beecheyi, Wheeler, Douglas et Evans (1941) ont recueilli sur l'oiseau, 70 Echidnophaga gallinacea et les ont inoculées à um cobaye qui mourut ensuite de peste. C'était la première fois qu'un 
oiseau était démontré porteur de parasites pesteux. Brown (1944) opérant de la même façon, au cours d'une épizootie de $C$. richardso$n i$, n'a pas pu mettre l'infection en évidence dans les 84 puces récoltées.

En plus d'E. gallinacea, les espèces récoltées dans le terrier de ce hibou sont $C$. idahoensis et Rectofrontia fraterna.

Eskey et Haas ont remarqué que les puces ont une spécificité marquée pour un hôte ou un groupe restreint. Cependant, des échanges de parasites sont fréquemment observés entre rongeurs d'espèces différentes.

Les recherches dans les terriers ont montré que certaines puces abandonnent volontiers leurs hôtes pour rester dans les nids, ce qui explique le petit nombre d'exemplaires trouvé sur certains rongeurs. Il ne semble pas que ces puces de terriers puissent subsister très longtemps en l'absence de rongeurs, car il n'en a pas été trouvé dans les terriers abandonnés.

Dans les expériences d'Eskey et Haas (1939), 31 espèces de puces ont pu s'infecter sur le cobaye. Elles seront citées plus bas dans un tableau. Chez 12 à $190 / 0$ des exemplaires, l'infection disparait au bout d'un certain temps, mais ce pourcentage est variable avec l'espèce ; il n'est que de $40 / 0$ pour $X$. cheopis.

L'intervalle entre l'infection de la puce et la transmission au cobaye varie de 5 à 130 jours.

Voici les espèces de puces selvatiques les plus remarquables en Amérique du Nord :

Ceratophyllus (= Diamanus) montanus (Baker). Très fréquente sur certains Citelles. Nous avons vu que, dans les expériences de Wheeler et Douglas, l'espèce s'est comportée, pour la souris blanche, comme un excellent vecteur, nettement supérieur à $X$. cheopis. Dans le travail d'Eskey et Haas, $250 / 0$ s'infectent en piquant une seule fois le cobaye septicémique. Une puce sur douze a transmis l'infection au cobaye et dans une autre série d'expériences, deux exemplaires infectent trois cobayes. Douglas et Wheeler (1943) ont établi que $600 / 0$ de $D$. montanus infectées peuvent libérer leur tractus digestif du bacille pesteux après un repas unique. Ce chiffre n'est que de $20 / 0$ pour $X$. cheopis.

Les bacilles restent virulents très longtemps dans les déjections ; celles-ci, au bout de deux mois, entrainent encore constamment la mort du cobaye.

Hoplopsyllus anomalus (Baker), est, avec la précédente, le principal parasite des Citelles réputés les plus dangereux. Elle se trouve assez souvent sur des genres différents : Neotoma, Cynomys... Evans, Wheeler et Douglas (1943) l'ont trouvée spontanément infectée, elle 
pique l'homme. Son pouvoir pestigère est discuté. Il est très faible pour Wheeler et Douglas (1941 et 1945) qui lui attribuent un « vector efficiency $\gg$ nul ; Eskey et Haas (1938 et 1939) ont cependant réussi la transmission au cobaye et des essais de transmissions faits en prenant pour sujet d'expérience un des hôtes habituels seraient désirables.

Ceratophyllus (= Thrassis) pandoræ (Jellisson); est l'espèce la plus abondante sur les Citelles de Wyoming et d'Oregon ; (Jellison, 1937), Eskey et Haas ont infecté 58 exemplaires et avec un lot de 6 de ceux-ci ont pu faire la transmission à 15 cobayes.

Ceratophyllus (= Opisocrostis) labis (J. et R.), puce de Citelle, a été trouvée infectée spontanément par Prince (1943). 178 exemplaires se sont infectés dans les expériences d'Eskey et Haas, et 14 puces infectées ont effectué la transmission à 35 cobayes.

Ceratophyllus (= Oropsylla) rupestris (Jord.), sur C. richardsoni et Mustela. Ozburn (1944) la suppose importante. Eskey et Haas ont obtenu trois infections expérimentales : deux puces ont infecté six cobayes.

Ceratophyllus (= Thrassis) francisi (Fox), sur C. beldingi, 21 infections expérimentales, 4 exemplaires infectent 8 cobayes.

Ceratophyllus (= Thrassis) arizonensis (Baker), sur Citelles, 58 infections expérimentales, 3 exemplaires infectent 5 cobayes.

Ceratophyllus (= Opisocrostis) tuberculatus (Baker), sur Citelles et chiens de prairies, 10 infections expérimentales, 1 exemplaire infecte 1 cobaye.

Ceratophyllus (= Opisocrostis) hirsutus (Baker), sur chiens de prairies, 70 infections expérimentales, 3 exemplaires transmettent à 4 cobayes.

Ceratophyllus $=$ (Thrassis) acamantis (Roths.) est la plus commune, à l'intérieur de la Colombie Britannique, sur les marmottes (Mail et Holland, 1942), 8 infections expérimentales, 1 exemplaire infecte 1 cobaye.

Ceratophyllus (= Thrassis) howelli (Jord.), sur marmottes, 6 infections expérimentales, 1 exemplaire infecte 2 cobayes.

Ceratophyllus (= Monopsyllus) eumolpi (Roths.), sur Eutamias, 31 infections expérimentales, 2 exemplaires infectent 4 cobayes.

Ceratophyllus (= Orchopeas) sexdentatus (Baker), sur Neotoma, 81 infections expérimentales, 3 exemplaires transmettent à 4 cobayes.

Quelques espèces s'ajoutent encore à la liste donnée par Eskey et Haas :

Ceratophyllus (= Thrassis) bacchi (Roths.), sur Citellus richardsoni et $C$. tridecimlineatus, 10 exemplaires s'infectent sur cobaye et 4 transmettent l'infection au cobaye (Prince, 1943). 
L'infection spontanée a été décelée au North Dakota. L'intervalle entre le repas infectant et la constatation de la transmission au cobaye a été de 23 à 34 jours.

Ceratophyllus (=Opisocrostis) bruneri (Baker), sur Citellus franklini, C. tridecimlineatus en Nebraska, 8 exemplaires s'infectent expérimentalement. 4 transmettent l'infection au cobaye. L'intervalle entre l'infection de la puce et la transmission au cobaye a été de 21 à 34 jours (Prince 1943).

Malareus telchinum (Roths.) est une puce de Microtus. Stewart (1940) pensait que c'était un des vecteurs les plus importants entre animaux domestiques et animaux sauvages, car elle a été trouvée sur les deux.

Dans les expériences d'Eskey et Haas, 74 exemplaires se sont infectés, mais c'est la seule, parmi les 15 espèces différentes étudiées, qui n'a pas pu infecter le cobaye ; Wheeler et Douglas (1941) ont abouti aux mêmes conclusions. Burroughs (1944) a pu infecter la puce sur Peromyscus maniculatus, Microtus californicus et sur la souris blanche. Il a mis en évidence le pouvoir pestigère de l'espèce sur Microtus californicus.

Cet exemple démontre qu'il est nécessaire de travailler avec l'hôte principal de la puce avant de conclure à la non-efficience de l'insecte. Et cela à fortiori, quand il s'agit de comparer quantitativement le pouvoir de transmission de deux espèces pulicidiennes différentes.

\section{Amérique du Sud}

En Amérique du Sud, la peste des rongeurs sauvages a été signalée en $1905,1924,1928,1932,1934$, et presque chaque année depuis cette date. Elle y semble établie de façon permanente, mais de manière plus ou moins bruyante, apparaissant en hiver, s'étendant sur de très vastes étendues, disparaissant au printemps. Les Caviidés et les Cricetidés sont spécialement atteints. Malgré la violence des épizooties, les cas humains sont rares. On constate parfois une infection animale de type subaigu, sans lésions macroscopiques.

Vioici les rongeurs particulièrement incriminés :

1. - Cavia aperea a été trouvé pesteux au Brésil, en Argentine (Uriarte, 1927) et en Equateur (Eskey, 1930).

2. - Microcavia australis a été trouvé infecté en Argentine. L'animal évite le contact humain. Il est parasité par différentes espèces des genres Parapsyllus, Rhopalopsyllus et Hectopsyllus. 
3. - Galea musteloides, trouvé infecté en République Argentine en 1937. C'est un Caviidé qui fuit l'homme et ne pénètre dans les habitations que quand elles sont abandonnées (J.-M. de la Barrera, 1937). Il est l'hôte de Rhopalopsyllus byturus.

4. Graomys griseoflavus est un cricetidé qui habite le creux des arbres mais peut envahir les habitations. En Argentine, il a été trouvé infecté en 1937 (De la Barrera) ; il est parasité par Rhopalopsyllus occidentalis et Craneopsylla wolffhuegeli.

5. Hesperomys muryllus, trouvé infecté dans les épidémies de la Pampas (Sussini, 1938).

6. Sylvilagus brasiliensis gibsoni, léporide des régions montagneuses (Sussini, 1938).

Citons enfin Monodelphis domesticus que Macchiavello (1941) soupçonne de transmettre la peste de rongeurs domestiques à rongeurs sauvages.

Les siphonaptères qui ont fait l'objet d'études expérimentales sont :

Parapsyllus talis (Jord.), avec lequel une expérience de transmission sur Microcavia australis a été positive. C'est l'espèce la plus fréquente sur les Caviides de République Argentine.

Hectopsylla suarezi (Fox) se trouve sur cobayes et lapins et joue un rôle dans certains cas comme vecteur entre les cobayes et l'homme (Suarez, 1942).

Rhopalopsyllus cavicola (Weig.) se trouve en Argentine et Equateur sur les. Caviides et parfois dans les vêtements et literies des Indiens. Le pouvoir pestifère a été vérifié expérimentalement, mais non le pouvoir pestigère (Long, 1935).

Rhopalopsyllus bohlsi var. jordani (Costa Lima), associée à une espèce du même genre non déterminée, est probablement le vecteur de la peste de rongeurs dans certaines parties du Vénézuéla et peut être responsable de la transmission à l'homme (Hecht, 1942).

\section{Russie}

Nous n'aurons en vue, dans ce chapitre, que la peste selvatique de la Russie occidentale (la Transbaïkalie sera étudiée en même temps que la Mongolie). Il existe deux foyers distincts :

- l'un au Sud-Est, dans les steppes argileuses, avec le Citellus pygmaeus comme principal réservoir de virus ;

- l'autre dans les zones sablonneuses du Sud où les rongeurs sont Mus musculus et Pallasiomys meridianus accompagnés de plusieurs autres espèces. 
La peste des Citelles a été découverte en 1912 (Deminsky, Berdnikov, cités par R. Jorge, 1928). L'épizootie est généralement à son maximum au mois de mai et juin, au début de la vie indépendante de la nouvelle génération. Les Citelles sont plus sensibles à la peste à cette époque. Pendant l'hibernation, au contraire, ils sont réfractaires, ou font une forme de peste chronique qui perpétue le germe d'une année à l'autre.

A un moindre degré, les puces qui se trouvent dans les nids de Citelles sont aussi susceptibles de conserver le bacille pesteux pendant l'hiver (Golov et Ioff, 1926), (Evseeva et Firsov, 1932).

La maladie humaine éclate au moment du maximum de l'épizootie des Citelles, au mois de mai et juin.

La peste des Souris et des Gerbilles a été mise en évidence en 1915 (Koltzof, Nikanoroff, cité par R. Jorge, 1928). Ici il n'existe pas de formes chroniques pour maintenir l'infection. Il se produit des épizooties massives qui réduisent la densité de la population murine, pour un certain temps ; puis une pullulation nouvelle des rongeurs entraîne de nouvelles épizooties. Le germe semble venir de la région intermédiaire, où Citelles et Souris coexistent et où les échanges de puces sont fréquemment observés entre les hôtes. Tikhomirova, Zagorskaia et Jiyine (1935) considèrent, au cuntraire, que la peste existe sous forme endémique dans la région sablonneuse et que l'échange des puces dans la zone intermédiaire donne des poussées épidémiques de peste chez les Citelles des steppes.

L'infection, chez l'homme, se voit en général en autonıne, aı moment de la récolte d'une graminée (Agriophyllum arenarium) qui sert de gîte et de nourriture aux souris.

La biologie des rongeurs a fait l'objet d'importants travaux :

1. Citellus pygmaeus (C. musicus et C. mugosaricus sont synonymes). - La reproduction se passe aussitôt après le sommeil hibernal, les femelles mettent bas au début de mai et allaitent un mois. Quand les petits sont âgés de 5 semaines, la mère les quitte et va se creuser un autre terrier pour l'hiver. Un des petits reste dans l'ancien terrier, les autres vont s'en creuser d'autres. Ils tombent en sommeil quand la température de l'air extérieur est de 3 à $4^{\circ} \mathrm{C}$. Ils sont diurnes et ne.se montrent pas, tant que l'herbe est mouillée. Leurs principaux ennemis sont les furets (Lebedev, 1925). La sensibilité maxima à la peste se trouve chez les jeunes au moment de leur migration, ce qui amène par suite des contacts plus fréquents une très forte mortalité $(850 / 0)$, pendant la première année de la vie (Kalaboukov et Zaievski, 1936). Il a été trouvé des puces et des tiques (Rhipicephalus schulzei), infectées de peste, même dans les terriers abandonnés (Golov et Kniazevski, 1930). 
Ceratophyllus tesquorum, Neopsylla setosa et Ctenophtalmus breviatus sont les principaux siphonaptères parasites.

Des coléoptères, de la famille des staphylinides, vivent dans les terriers de Citelles et se nourrissent, en partie tout au moins, de puces ou de larves de puces. Il sont certainement d'utiles auxiliaires dans la lutte contre la peste selvatique (Flegontova, 1938).

2. Cynomys fulvus. - Le suslik s'oppose au précédent en ce qu'il vit dans la zone sablonneuse, mais sa biologie est proche de celle de Citellus pygmaeus. Comme celui-ci, il a des terriers d'hiver, profonds, et des terriers d'été, plus superficiels. Il se déplace volontiers lorsqu'il est jeune, ou lorsqu'il recherche des régions couvertes d'herbes fraîches. L'hibernation cesse en général à la fin du mois de mars. Ses parasites sont : Oropsylla ilovaiski, Ctenophtalmus breviatus, C. orientalis, C. pollex, Ceratophyllus tesquorum et Neopsylla setosa.

3. Pallasiomys meridianus. - Bien connu sous le nom de Mérion, il est le principal habitant des steppes sablonneuses du Sud de la Volga et de l'Oural. L'animal entre en contact avec l'homme car, en septembre-octobre, il amasse une réserve de grains qu'il cache le plus souvent dans les huttes de paysans. Le Mérion ne porte que peu de puces, car, d'une part, il les détruit, et, d'autre part, il change souvent de nids, en raison des déplacements de sable. Un fait curieux est mentionné : chez les Mérions apparemment sains, sacrifiés 20 jours à 1 an après leur inoculation, l'autopsie et l'examen bactériologique révèlent la présence de bacilles pesteux dans les organes. Les puces du rongeur sont : Ceratophyllus mokzreckyi, C. læviceps et Xenopsylla conformis (Tikhomirova, 1934).

4. Dipus sagitta. - Cette Gerboise passe la journée dans des terriers sur des plateaux de sables arides, mais la nuit elle est très vagabonde. Son sommeil dure de novembre à mars. Elle s'installe souvent dans le voisinage des maisons et les chats en apportent parfois les cadavres dans les habitations. Des familles entières succombent à la peste. Expérimentalement, l'animal est très sensible, et peut servir de test pour des doses minimes (Tikhomirova, 1935). Les puces Ophtalmopsylla kasakiensis, O. volgensis, Mesopsylla levis, $M$. Iuschkan sont propres aux Gerboises. On a trouvé également sur elles : $X$. mycerini et $C$. læviceps.

5. Ellobius talpinus vit dans une région de terres noires, dans les parties les plus basses de la steppe sèche. Pendant les chaleurs de l'été, la population de la steppe environnante se concentre dans ces régions. Citellus pygmaeus, en particulier, s'y réfugie, et contamine Ellobius talpinus (Gaisky, 1931). La puce la plus fréquente est Xenopsylla mycerini. Ioff (1935), donne la liste suivante : X. magda- 
linæ, C. tesquorum, Amphipsylla rossica, Neopsylla setosa, Ctenophtalmus orientalis, C. breviatus, C. pollex, C. gigantospalacis. Il taut y ajouter un Ixode, Rhipicephalus schulzei.

6. Lagurus lagurus. - Souris de champs trouvée infectée par Kniazevsky et Grichina (1928). Dans les nids de la souris ont été trouvés Ctenophtalmus breviatus, C. læviceps, Amphipsylla rossica, Ceratophyllus mokzreckyi et Rhadinopsylla sp. Zasukhin et Tiflov (1936) ont donné une liste plus complète des ectoparasites.

7. Rhombomys opimus. - Semble le réservoir de virus dans le Turkestan et la région transcaspienne (Nikanoroff et Knizaevsky, 1927). Les ectoparasites sont : Ctenophtalmus dolichus, Ophtalmopsylla volgensis, Rhadinopsylla cedestis; Ceratophyllus du groupe laviceps, Xenopsylla du groupe conformis, Coptopsylla proche de lamellifer et Neopsylla proche de setosa.

8. Mus musculus, Mus musculus wagneri et Mus tamaricinus doivent être considérés en Russie comme des rongeurs semi-selvatiques, s'associant à Pallasiomys meridianus dans les épizooties et échangeant fréquemment avec lui leurs parasites : C. mokzreckyi, C. læviceps, X. mycerini. Une liste des ecto-parasites a été donnée par Zasukhin et Tiflov (1936). La puce propre à la souris, Leptopsylla musculi se fixe peu volontiers sur l'homme; en outre, puisque les souris ont, le plus souvent, un faible degré d'infestation, elles sont, en réalité moins dangereuses qu'il ne pourrait le sembler à première vue.

9. Microtus arvalis participe aussi aux foyers des zones sablonneuses. Ses ecto-parasites sont : Ctenophtalmus orientalis, C. assimilis.

10. Putorius eversmanni chasse les Citelles et les Gerboises et peut être infecté par les puces de ses victimes et contracter la peste (Ioff, 1929). Il est remarquable qu'il héberge une puce très proche de la puce humaine, Pulex irritans, var. fulvus, qui est très fréquente. Dix autres espèces de puces ont été trouvées sur lui.

11. Oenanthe oenanthe. - Cet oiseau, qui peut fréquenter des terriers de rongeurs, construit son nid dans les maisons. Il est susceptible de transporter des puces de rongeurs : C. tesquorum, Ctenophtalmus breviatus, et Neopsylla setosa (Sergeev, 1937).

Les arthropodes les plus intéressants sont :

\section{Chez les Sciurida}

Ceratophyllus tesquorum (Wagn.), dont Citellus pygmaeus est le principal hôte, mais il en change volontiers. Il garde la peste pen- 
dant l'hiver (Evseeva, Firsov, 1932) et expérimentalement l'infection a même pu persister 275 jours (Golov et Ioff, 1926). Sa grande abondance (94 $0 / 0$ sur les Citelles), la facilité avec laquelle il attaque l'homme, ont amené Ioff et Pokrovskaya (1929) à le considérer comme le vecteur le plus dangereux en Russie (son maximum d'abondance, en mai-juillet, correspond aux épizooties). Cependant, à cause de la chaleur et de la sécheresse des steppes, il ne semble pas vivre longtemps hors de son hôte.

On connaît deux variétés : C. tesquorum jennissejensis sur $C$. cyersmanni et $C$. tesquorum mongolicus sur $C$. mongolicus.

Tinker et Zenkevitch (1934) remarquent que cette puce, ainsi que Neopsylla setosa, ont tendance à sortir spontanément du terrier la nuit. Les deux espèces résistent peu quand elles sont exposées au soleil.

Neopsylla setosa (Wagn.), est associée à l'espèce précédente sur les Citelles. L'infection spontanée a pu être trouvée par Tumansky et Poliak (1931) et retrouvée plusieurs fois depuis cette date. L'infection persiste expérimentalement 180 jours (Golov et Ioff). Aristarkhova (1930) a prouvé que cet insecte était capable expérimentalement de transmettre la peste. Il a tendance à rester dans les terriers, mais est capable de piquer l'homme.

Frontopsylla semura (Wagn. et Ioff). - L'infection persiste expérimentalement 203 jours (Golov et Ioff).

Ctenophtalmus breviatus (Wagn. et Ioff), ne pique pas l'homme, même à jeun. L'infection expérimentale persiste 296 jours.

Ctenophtalmus pollex attaque l'homme. L'infection spontanée a été découverte par Tumansky et Poliak (1931). L’infection expérimentale persiste 203 jours.

Ctenophtalmus orientalis. - Ces trois dernières espèces sont moins fréquentes sur les Sciuridés. Ce sont essentiellement des parasites de Microtinés. Elles ont été étudiées également pour la tularémie, maladie dont elles sont parfois spontanément infectées.

\section{Chez les Murina}

Ceratophyllus mokzreckyi (Wagn.), est une puce de Souris, mais se trouve aussi sur Gerboises et Susliks. C'est un vecteur expérimental (Golov et Ioff, 1926), néanmoins l'infection spontanée a été mise en évidence par Tikhomirova et ses collaborateurs (1935). Elle se rencontre quelquefois sur l'homme.

Ceratophyllus consimilis, également puce de Souris, qui pique l'homme. C'est un vecteur expérimental ; elle reste infectieuse 210 jours de 0 à $15^{\circ} \mathrm{C}$. 
Ctenophtalmus orientalis.

Leptopsylla musculi.

\section{Chez les Dipodidæ}

Ophtalmopsylla volgensis.

O. kasakiensis.

Mesopsylla hebes.

M. lenis.

M. tuchkan.

\section{Chez les Microtinæ}

Ctenophtalmus breviatus.

C. pollex.

C. orientalis.

Ceratophyllus tesquorum.

C. consimilis.

Amphipsylla rossica.

\section{v. Chez les Cricetinæ}

Différents Ctenophtalmus.

\section{Chez les Gerbillinæ}

Ceratophyllus læviceps, dont Golov et Ioff ont observé le bloquage du proventricule.

Xenopsylla mycerini.

En plus des siphonaptères, un certain nombre d'arthropodes ont été étudiés en Russie :

$1^{\circ}$. Argas persicus. - $230 / 0$ s'infectent sur cobayes septicémiques. L'injection de l'Argas broyé à la souris a été encore positive 110 jours après le repas (Faddeeva, 1932).

$2^{\circ}$ Dermacentor silvarum. - Les larves et nymphes nourries sur cobayes infectés ont permis d'isoler la peste des larves après 2, 4, 6 et 10 jours, et des nymphes après 2 à 6 jours ; mais les bacilles n'ont jamais été retrouvés dans les ixodes adultes, le virus se perd à chaque mue (Zasukhin et Tikhomirova, 1937).

$3^{\circ}$ Hyalomma volgense. - Depuis l'état larvaire, jusqu'au stade adulte, elle peut s'infecter en se nourrissant sur des animaux pesteux et héberger dans son organisme des hacilles virulents. Ils restent virulents pendant au moins 11 jours chez l'adulte, 3 jours 
chez la nymphe et 7 jours chez la larve. La piqûre directe d'une tique pesteuse peut infecter l'animal sain et provoquer la mort. Elle peut donc jouer un rôle dans la propagation de la peste (Borgzenkov et Donskov, 1933).

$4^{\circ}$ Rhipicephalus schulzei. - Fréquent sur les Citelles, mais se trouve aussi sur beaucoup d'animaux domestiques, moutons, chiens, chameaux (Pavlovsky, 1931). Des échantillons ont été trouvés infectés dans des nids abandonnés de Citellus pygmaeus (Golov et Kniazevski, 1930).

\section{Asie}

La peste selvatique sévit sur d'immenses territoires, en Transbaikalie, dans les steppes mongoles et en Mandchourie. On peut la limiter approximativement par la Sibérie au Nord, le Kinghan à l'Est, le Thibet au Sud, l'Oural à l'Ouest.

Il est généralement admis que c'est là le foyer dont sont parties toutes les épidémies historiques. La nature pesteuse des épizooties a été démontrée en 1911 par Zabolotny (cité par Joyeux, 1944).

Le principal rongeur est Arctomys bobac, viennent ensuite plus accessoirement, Citellus dauricus et Alactaga mongolica.

$1^{\circ}$ Arctomys bobac. - C'est le classique «tarbagan ». Bjeliavski et Rjesketnikoff (cités par Wu Lien Teh, 1936), l'ont trouvé pesteux dès 1895. L'animal creuse des terriers profonds, il vit en petites ou en grandes colonies, chaque famille habitant un terrier séparé. Ils cohabitent au printemps, après la longue hibernation (d'octobre à avril). La femelle, fin mai, donne de 2 à 7 petits. Ils ne sont pas migrateurs. L'usage commercial que l'on fait de sa fourrure explique le danger qu'il représente pour les habitants de la région. Les parasites sont :

a) Ceratophyllus (= Oropsylla) silantiewi (Wagn.). - Elle a été trouvée spontanément pesteuse (Suknev et Suknev, 1927). C'est un vecteur expérimental pour le cobaye. Elle reste infectieuse 385 jours de 0 à $15^{\circ} \mathrm{C}$. Un trait caractéristique de l'espèce est la ténacité avec laquelle elle se maintient sur le corps de son hôte ; elle peut être trouvée sur les dépouilles de celui-ci, ce qui est un grand danger pour l'homme, car elle le pique facilement. Elle est plus fréquente en automne, au moment où l'homme entre en contact avec le «tarbagan » et où la maladie humaine est à son maximum.

b) Ceratophyllus tesquorum (Wagn.). - C'est aussi un parasite des Citelles, de Russie Occidentale, elle a été vue avec ceux-ìi. 
On signale aussi sur Arctomys bobac différents arthropodes. Deux Ixodes :

Rhipicephalus hrmaphysaloides ne pique pas l'homme, même après une mise à jeun de 3 semaines. Il reste cependant sur les dépouilles des animaux et pourrait jouer un rôle de résevoir passif du bacille.

Ixodes hexagonus, spontanément infecté (Tikhomirova et Nikanoroff, 1929), cité par Wu Lien Teh (1936).

Un pou :

Linognatoides citelli. - Se rencontre sur les chasseurs. L'insecte a été trouvé spontanément infecté sur Arctomys bobac. L'infection a persisté 13 jours. La transmission a été réalisée en plaçant, sur un Citelle, 40 poux récoltés sur un tarbagan, 40 heures avant sa mort.

$2^{\circ}$ Citellus dauricus est aussi victime des épizooties en Transbaikalie (Skorodoumoff, Bull. Off. Int. d'Hyp. Publ., 1929). Il est parasité par C. tesquorum. Non recherché pour sa fourrure, il vit à proximité des demeures humaines et peut être une source de danger.

$3^{\circ}$ Alactaga mongolica, a été trouvé infecté par Skorodoumoff (1929). Elle héberge en Mandchourie une sous-espèce de $C$. tesquorum, et elle est considérée comme un réservoir de peste.

Ando, Kuranchi et Nishimura (1931) considèrent ces deux derniers rongeurs comme les plus importants en Mongolie intérieure ; mais il leur semble «plus raisonnable d'admettre que l'infection a été principalement propagée par l'homme », parasité par de très nombreux poux et puces. (Un pou recueilli sur le cadavre d'un pesteux a permis d'isoler le bacille de Yersin).

Aux Indes, et dans les Iles de la Sonde, il n'existe pas de peste selvatique, mais un certain nombre d'animaux sont contaminés par la peste des rats domestiques. Les espèces qui ont été trouvées infectées spontanément seront citées dans un tableau. Quelques espèces de siphonaptères ont été étudiées.

Ceratophyllus nilgiriensis se trouve sur Gunomys kok et d'autres rongeurs. On la soupçonne d'être associée à la peste dans certaines parties des Indes. Elle porte les bacilles pesteux dans le tube digestif 7 jours après un repas sur un hôte infecté, mais n'a pas transmis la peste à la souris (George et Timothy, 1941).

Stivalius $s p$. a transmis la peste à la souris blanche 3 jours après la séparation d'un hôte infecté ; elle a été trouvée sur Gollunda ellioti et Gunomys kok (George et Timothy, 1941). 


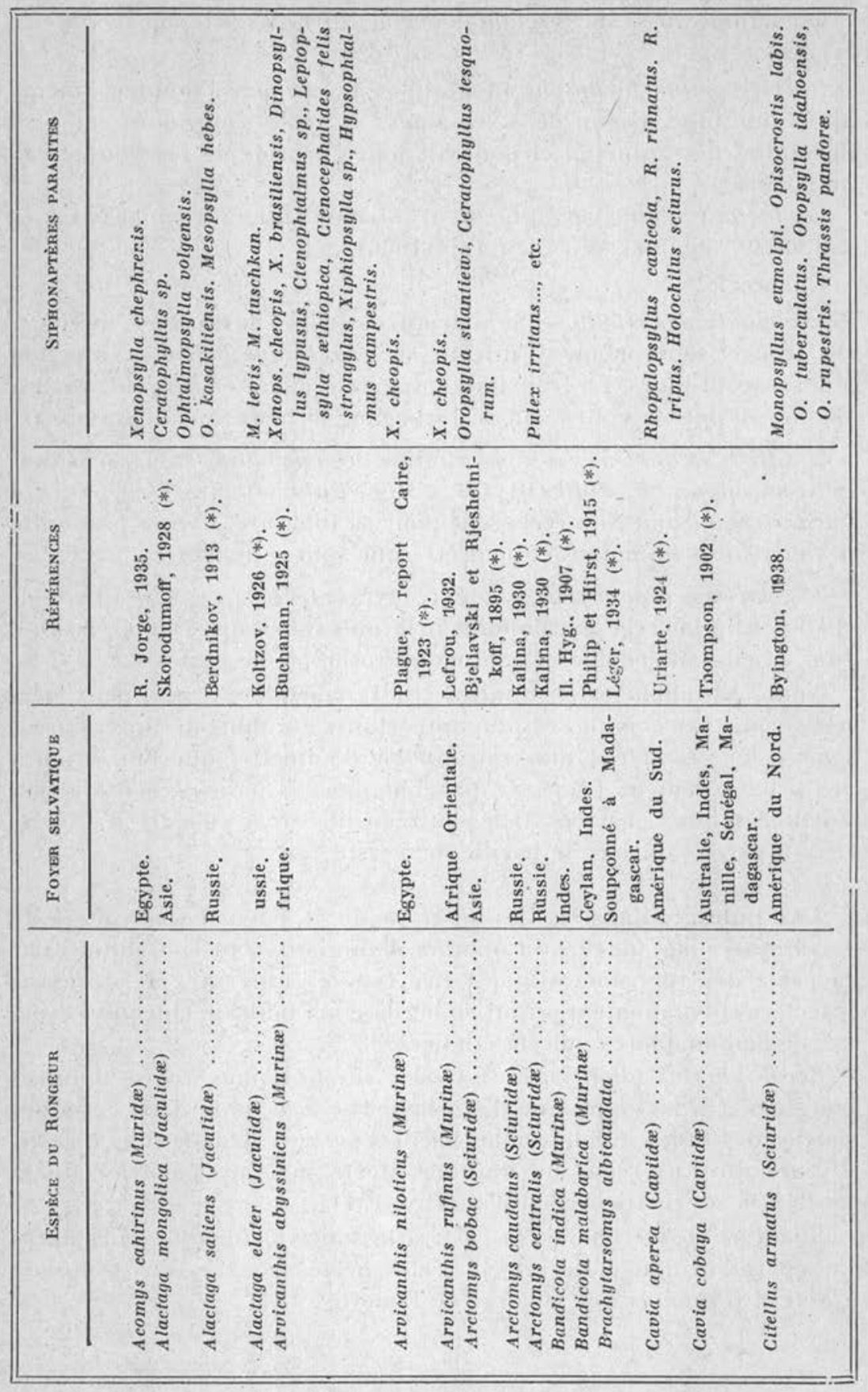




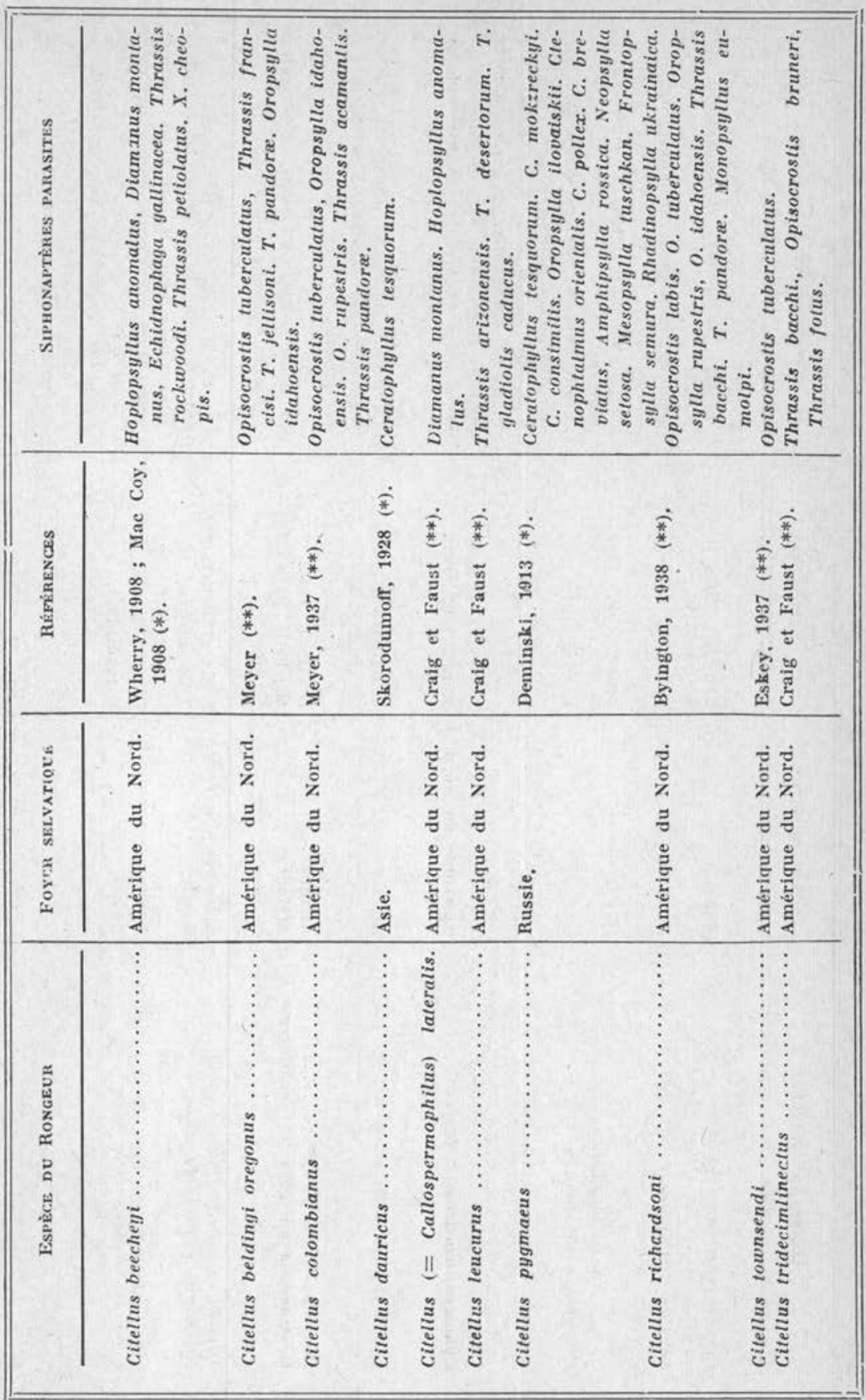

Ann, de Parasitologie, t. XXII, $x^{o s} 3-4,1947$. 


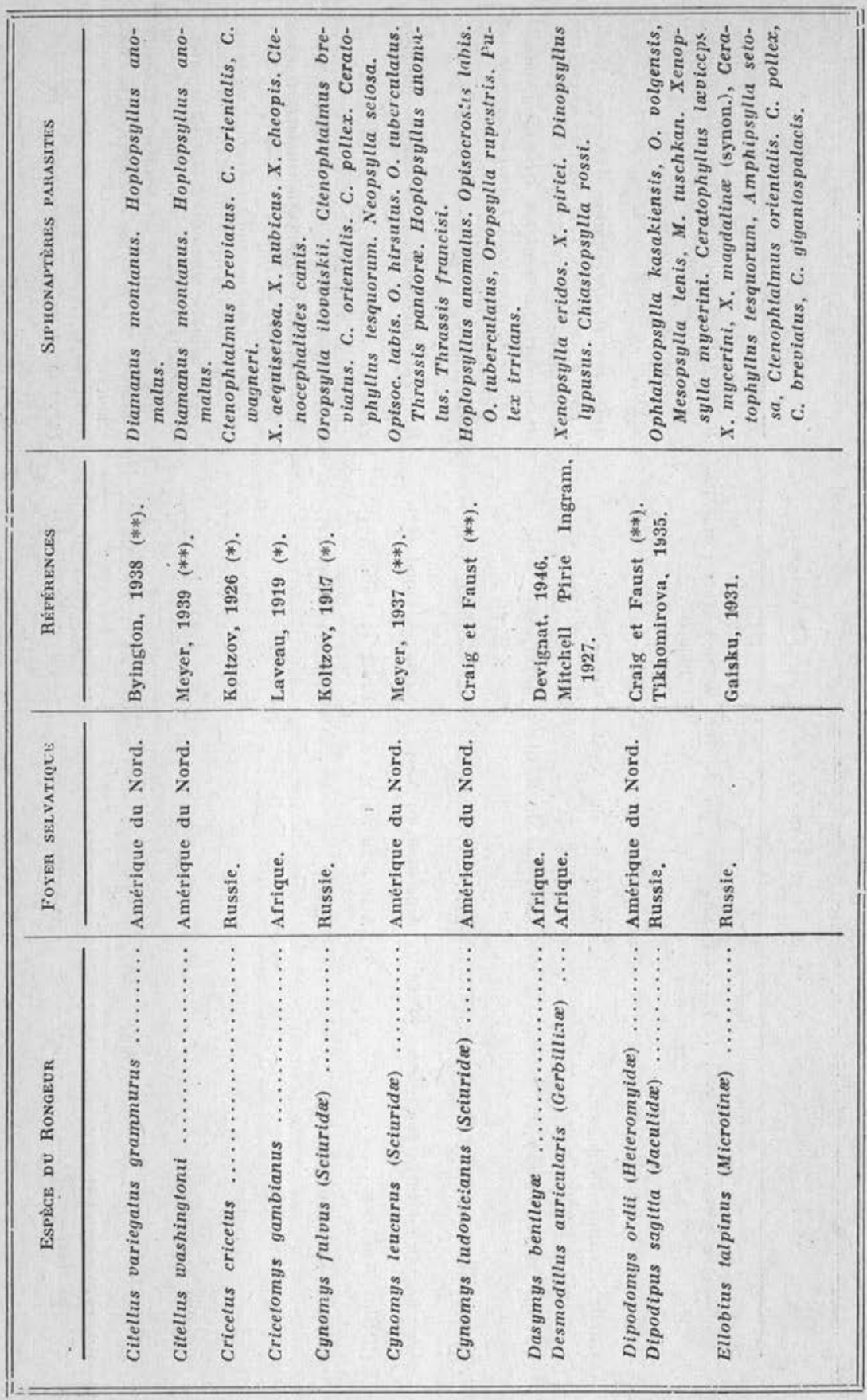




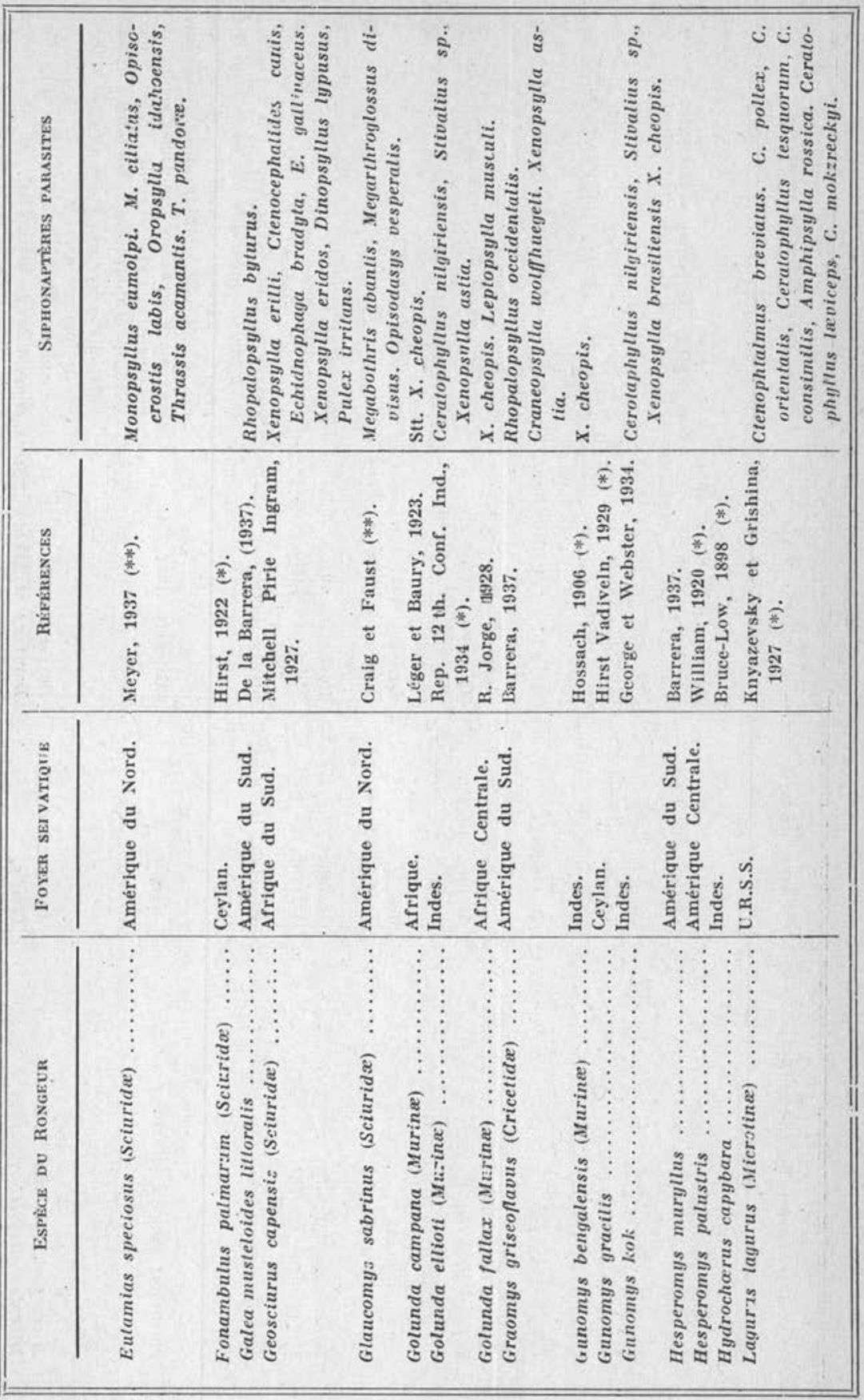




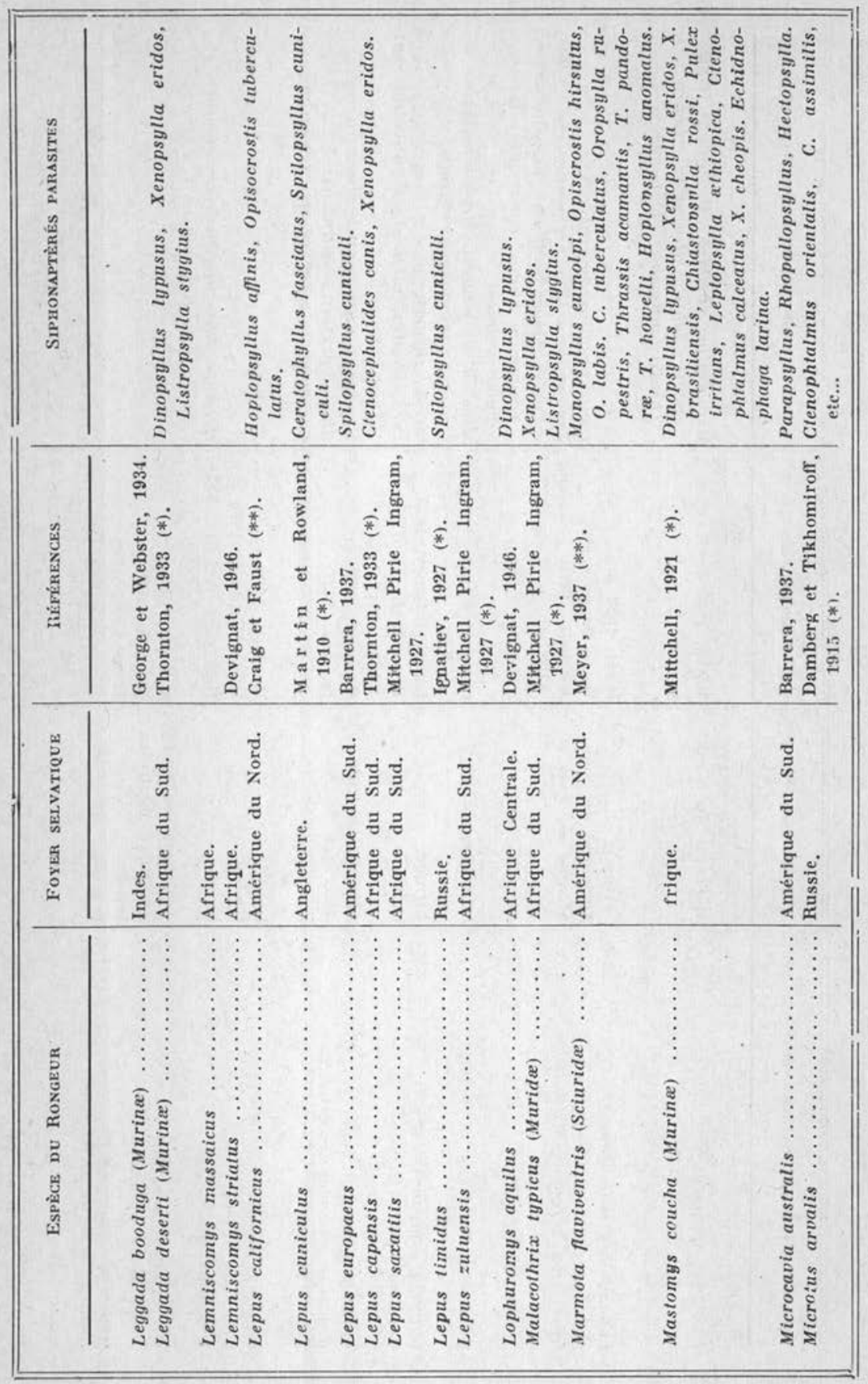




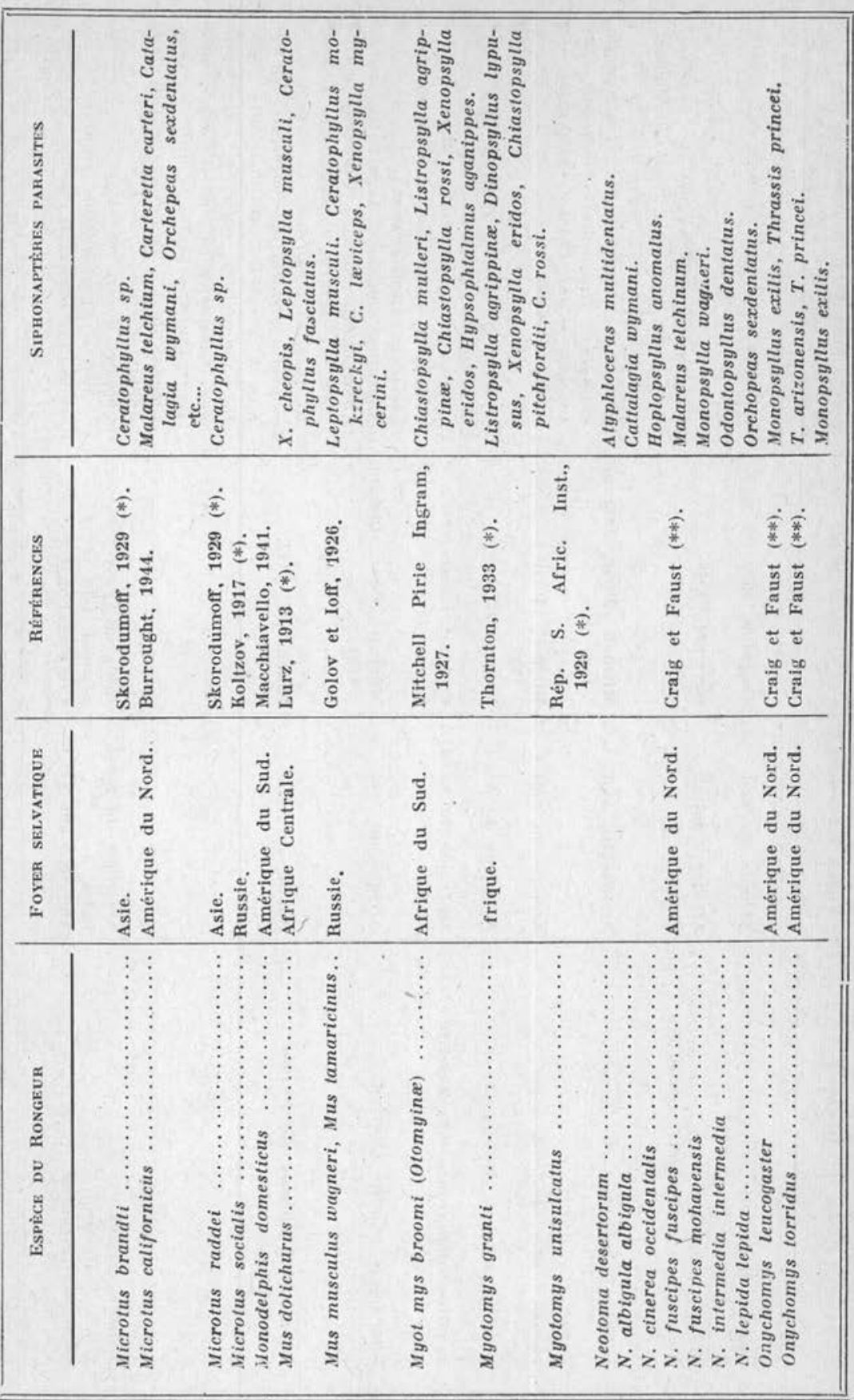




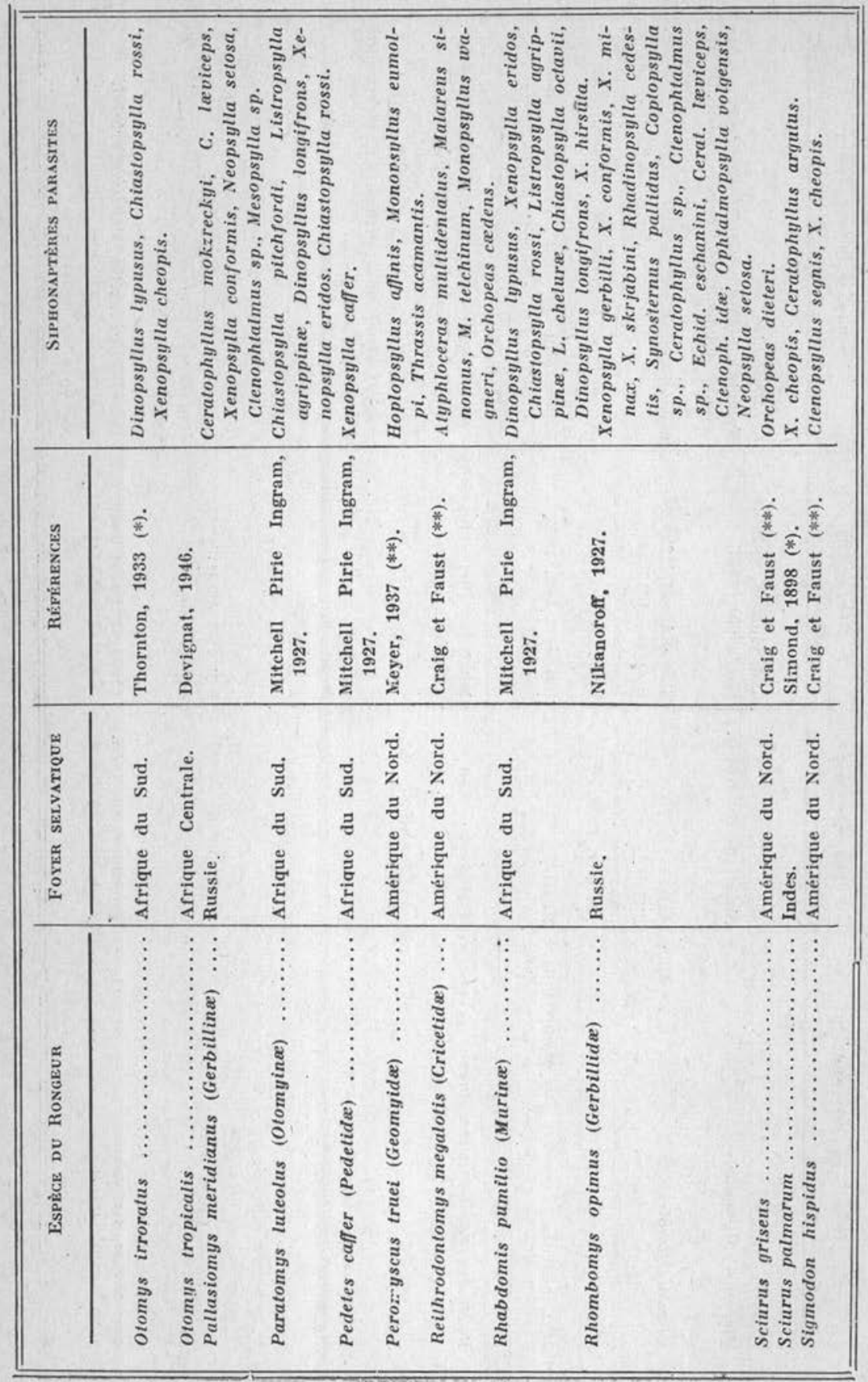




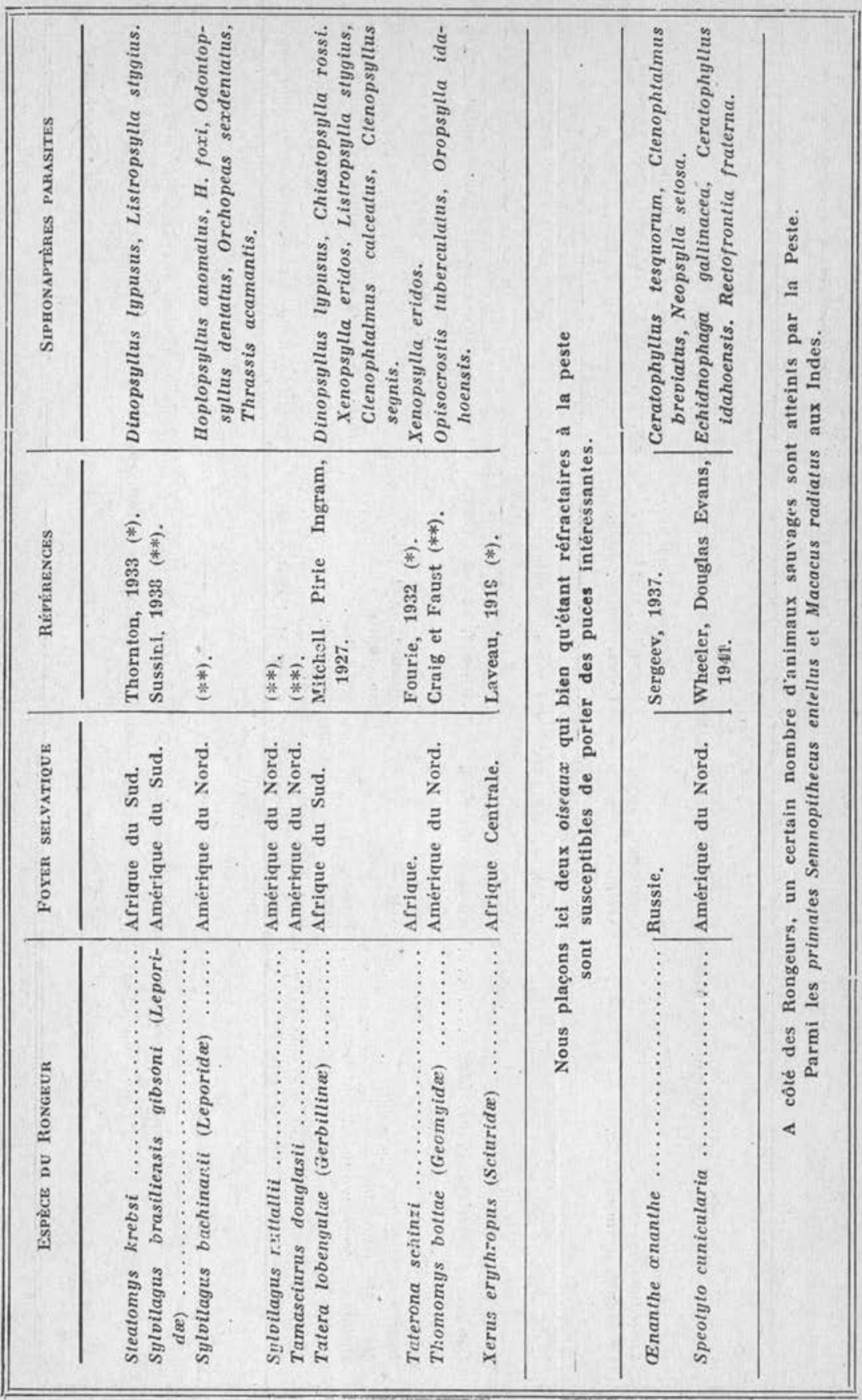




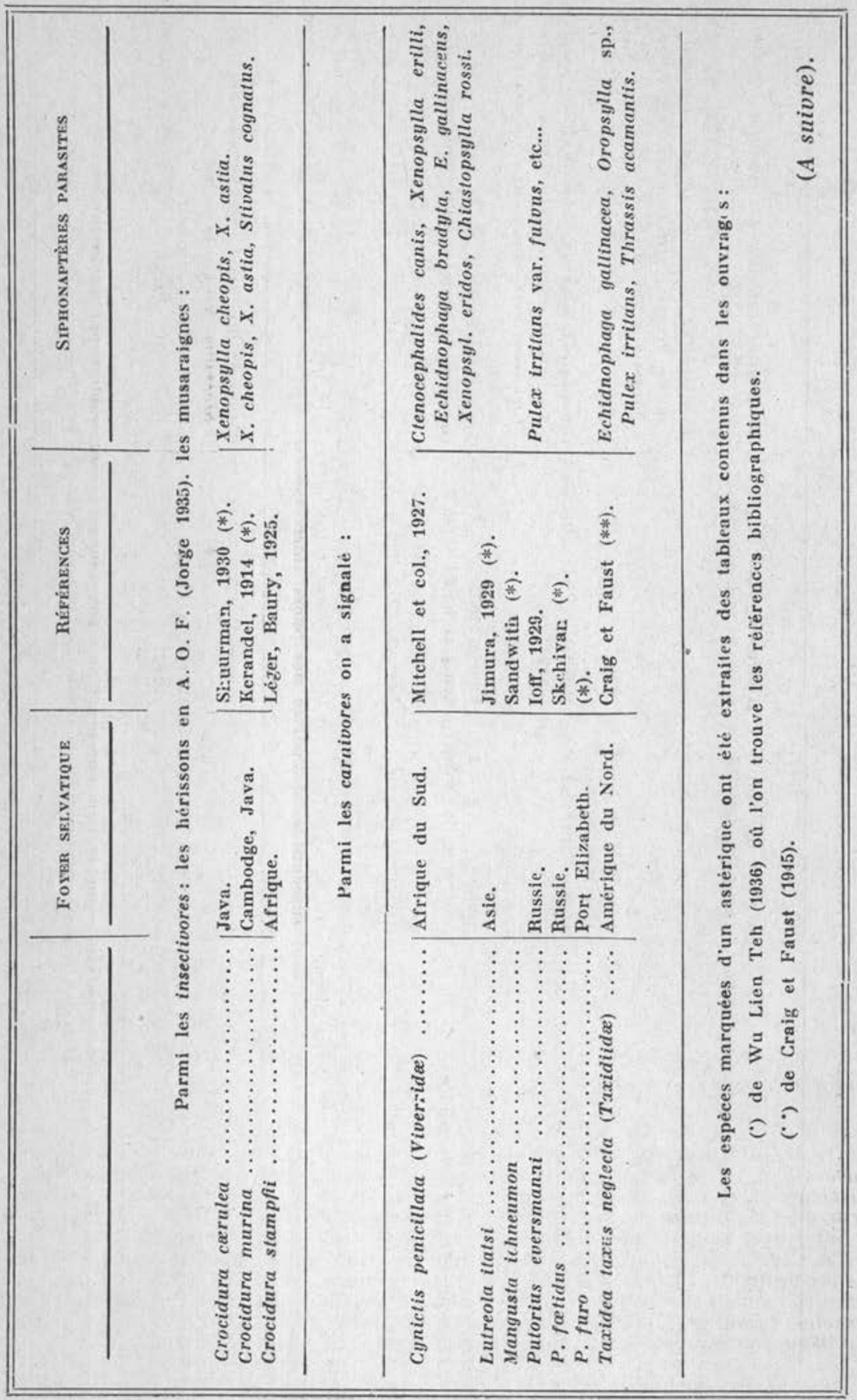

